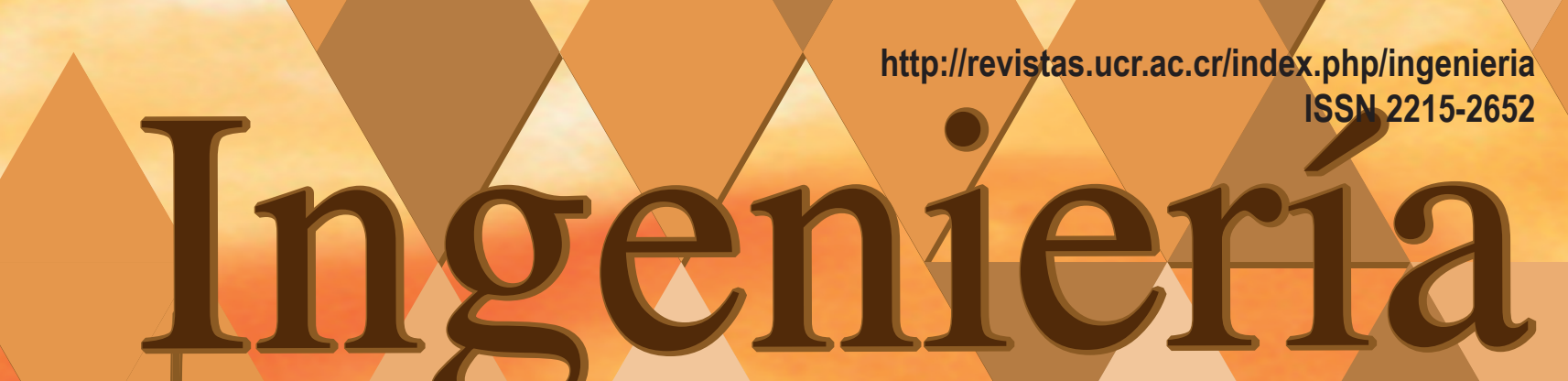

Revista de la Universidad de Costa Rica FEBRERO/AGOSTO 2014 -VOLUMEN 24 Número (1)

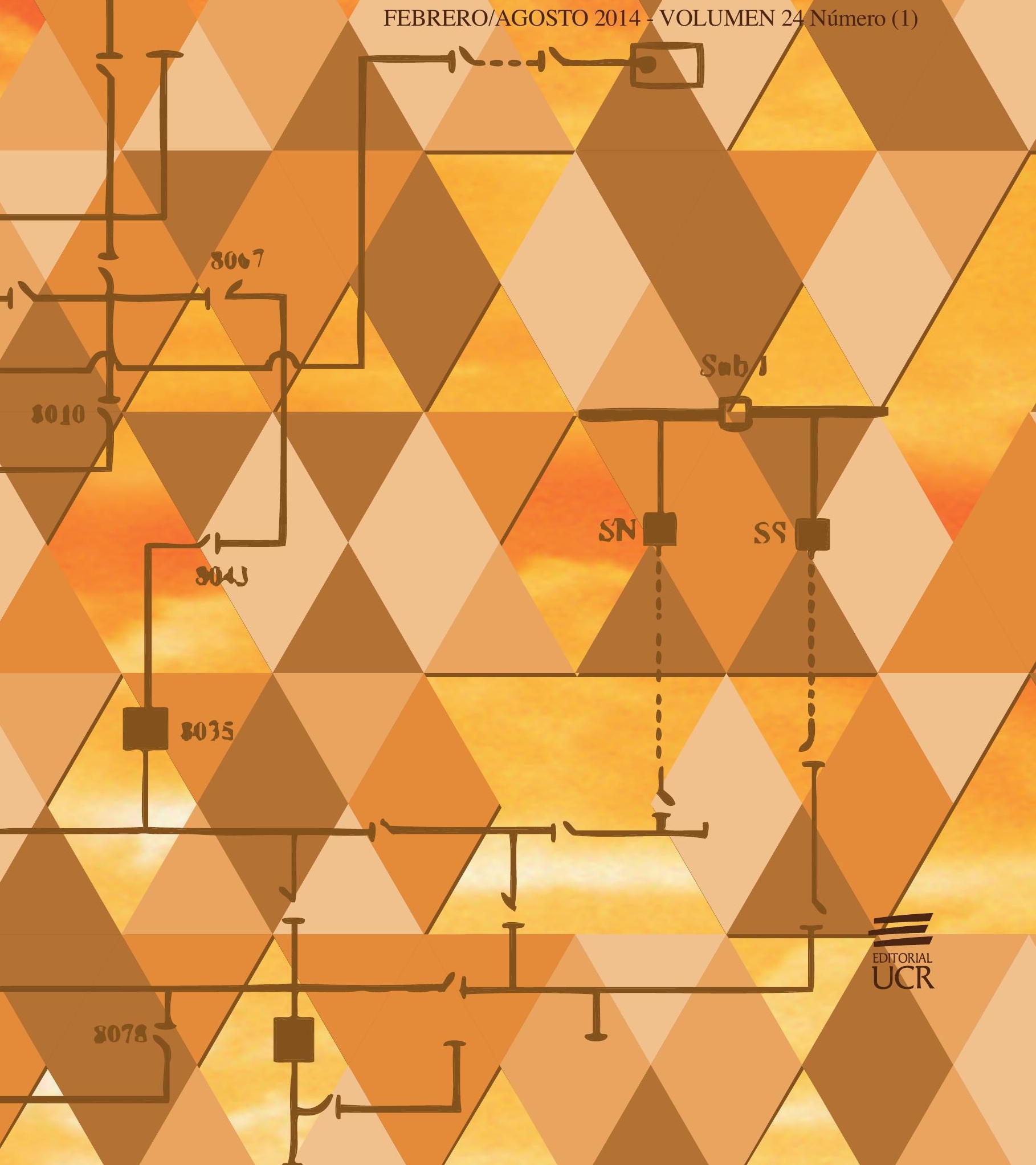




\title{
RECONFIGURACIÓN TOPOLÓGICA PARA LA REDUCCIÓN DE PÉRDIDAS DE ENERGÍA EN LA RED DE MEDIA TENSIÓN DE LA CNFL
}

Raúl Fernández Vásquez.

\begin{abstract}
Resumen
Inicialmente se realizó la caracterización y análisis de las pérdidas de energía que se dan en la red de media tensión de la Compañía Nacional de Fuerza y Luz (CNFL), concluyendo con la determinación de la constante que relaciona el factor de carga con el factor de pérdidas de los circuitos de distribución. Posteriormente, utilizando el método heurístico y la programación lineal, se desarrolló un programa en MATLAB que determina las pérdidas de energía en los circuitos de media tensión y las posibles combinaciones que un sistema tiene para intercambiar secciones mediante el cierre y apertura de interruptores y cuchillas, calculando las pérdidas para cada configuración y determinando así la topología con las menores pérdidas de energía. Los resultados de pérdidas obtenidos fueron validados con el software CYMDIST. Finalmente, se implementa el programa desarrollado en la zona oeste del área de servicio de la CNFL compuesta por cinco subestaciones y más de 129 millones de topologías posibles. La configuración con las menores pérdidas las reduce en $285 \mathrm{MWh}$ al año representando esto más de 15 millones de colones en ese periodo.
\end{abstract}

Palabras clave: distribución de energía, reconfiguración topológica, pérdidas de energía, operación de redes.

\begin{abstract}
This work deals with the characterization and analysis of energy losses in the medium voltage network of Compañía Nacional de Fuerza y Luz (CNFL). It determines parameters such as load factor, losses factor and the percentage they represent. It also includes the determination of the constant relating the load and losses factors of the distribution circuits of the CNFL. Using the heuristic method and linear programming, a program in MATLAB was developed to determine the energy losses in the medium voltage circuits and the possible combinations the system has, in order to change sections by closing and opening switchgear devices, calculating the losses for each configuration and thus, determining the topology with the lowest energy losses. After this, losses results are checked with CYMDIST, a distribution network simulation software. Finally, the program is implemented in the western service area of the CNFL, consisting of five substations and more than 129 million possible topologies. The best configuration found under the selected criteria reduces the losses by $285 \mathrm{MWh}$ per year, representing more than 30000 US dollar.
\end{abstract}

Keywords: distribution network, topology reconfiguration, energy losses, network operation.

Recibido: 16 setiembre de 2013 - Aprobado: 16 enero 2014

\section{INTRODUCCIÓN}

La topología de los alimentadores de media tensión de la CNFL se ha determinado a partir de un balance de carga entre los diferentes circuitos y entre las fases de los mismos. El cambio de cargas entre circuitos se ha dado producto de estos balances o por asuntos de atención de fallas o mantenimientos programados. Estos métodos sugieren que podrían existir topologías distintas a la actual que presenten menores pérdidas de energía y que son posibles de adoptar con tan solo el cierre y apertura de medios de conexión existentes.

Este análisis tiene implícito el problema de múltiples opciones que puede ser solucionado con el desarrollo de un programa reiterativo que determine las pérdidas para cada una de las posibles topologías. Este tipo de problema puede ser resuelto con distintos métodos, en este caso se utilizó el método heurístico y la programación 
Tabla 1. Pérdida de energía para los circuitos analizados.

\begin{tabular}{cccccc}
\hline Circuito & Factor de carga & \multicolumn{2}{c}{ Pérdida en demanda máxima } & Pérdida de energía & \\
& & $\mathbf{k W}$ & $\mathbf{\%}$ & $\mathbf{k W h}$ & $\mathbf{\%}^{\mathbf{1}}$ \\
\hline EL & 0,568 & 2,22 & 0,056 & 153 & 0,041 \\
FA & 0,682 & 8,51 & 0,144 & 749 & 0,111 \\
SJ & 0,621 & 122,51 & 0,689 & 9268 & 0,500 \\
IA & 0,706 & 51,76 & 0,495 & 5100 & 0,411 \\
IN & 0,745 & 32,58 & 0,259 & 3341 & 0,212 \\
AS & 0,635 & 56,80 & 0,517 & 4549 & 0,388 \\
PA & 0,619 & 64,54 & 0,586 & 4886 & 0,427 \\
MU & 0,610 & 18,74 & 0,202 & 1439 & 0,151 \\
SN & 0,694 & 78,69 & 0,779 & 7307 & 0,620 \\
TR & 0,647 & 15,27 & 0,226 & 1304 & 0,177 \\
BA & 0,730 & 62,47 & 0,566 & 6115 & 0,452 \\
GU & 0,766 & 35,96 & 0,403 & 3917 & 0,341 \\
RA & 0,657 & 52,65 & 0,406 & 4361 & 0,305 \\
PU & 0,619 & 47,44 & 0,380 & 3695 & 0,285 \\
LL & 0,684 & 91,19 & 0,575 & 8319 & 0,457 \\
SL & 0,627 & 135,37 & 0,946 & 10085 & 0,670 \\
Promedio & 0,663 & & 0,452 & & 0,347 \\
\hline
\end{tabular}

lineal por ser uno de los métodos más utilizados para resolver problemas de esta naturaleza.

A la fecha han sido publicados artículos relacionados con el uso de los métodos mencionados aplicados a redes hipotéticas como las establecidas por IEEE y calculadas con valores genéricos o típicos de la constante del factor de pérdidas. En este artículo se muestra el proceso seguido para determinar el valor de la constante del factor de pérdidas específico para la red en cuestión y se expone la aplicación de la reconfiguración a una red de media tensión real y los principales resultados obtenidos.

\section{PÉRDIDAS DE ENERGÍA EN CIRCUITOS DE MEDIA TENSIÓN}

De acuerdo a Kersting (2000), la energía perdida en un alimentador de media tensión está dada por la siguiente ecuación:

$E_{\text {perdida }}=P_{\text {prom }} \cdot t$

Donde, $\mathrm{P}_{\text {prom }}$ son las pérdidas promedio del alimentador (W) y $t$ el tiempo (h).

Las pérdidas promedio se obtienen mediante la siguiente ecuación 
Tabla 2. Factor A para los alimentadores de la CNFL.

\begin{tabular}{ccccc}
\hline Circuito & $\begin{array}{c}\text { Demanda } \\
\text { máxima(MW) }\end{array}$ & Factor de carga & $\begin{array}{c}\text { Factor de } \\
\text { pérdidas }\end{array}$ & Constante A \\
\hline EL & 3,98 & 0,568 & 0,413 & 0,369 \\
FA & 5,90 & 0,682 & 0,524 & 0,273 \\
SJ & 17,79 & 0,621 & 0,450 & 0,277 \\
IA & 10,46 & 0,706 & 0,587 & 0,426 \\
IN & 12,58 & 0,745 & 0,611 & 0,296 \\
AS & 10,99 & 0,635 & 0,477 & 0,317 \\
PA & 11,02 & 0,619 & 0,451 & 0,288 \\
MU & 9,29 & 0,610 & 0,457 & 0,358 \\
SN & 10,11 & 0,694 & 0553 & 0,336 \\
TR & 6,76 & 0,647 & 0,508 & 0,393 \\
BA & 11,05 & 0,730 & 0,583 & 0,254 \\
GU & 8,93 & 0,766 & 0,649 & 0,347 \\
RA & 12,96 & 0,657 & 0,493 & 0,275 \\
PU & 12,48 & 0,619 & 0,464 & 0,341 \\
LL & 15,48 & 0,684 & 0,543 & 0,348 \\
SL & 14,30 & 0,627 & 0,444 & 0,216 \\
Promedio & 10,904 & 0,663 & 0,513 & 0,319 \\
\hline
\end{tabular}

$P_{\text {prom }}=P_{\text {max }} \cdot F P$

Donde, $\mathrm{P}_{\max }$ son las pérdidas máximas en el alimentador (pérdidas para la máxima demanda del circuito) y $F P$ el factor de pérdidas.

El factor de pérdidas depende de la forma de la curva de demanda del alimentador en cuestión y está dado por la siguiente ecuación:

$F P=A \cdot F C+(1-A) \cdot F C^{2}$

Donde, $F C$ es el factor de carga del alimentador (demanda promedio entre demanda máxima) y A es la constante del factor de pérdidas que varía entre 0,15 y 0,3 según Short (2004).

Los cálculos realizados para la determinación de las pérdidas de los alimentadores de la CNFL y la constante del factor de pérdidas se realizaron con CYMDIST. Para realizar las simulaciones, este programa asigna a cada carga (transformador de distribución) la misma demanda porcentual de acuerdo a su capacidad nominal y a la demanda total del circuito ingresada por el usuario. Este supuesto es también utilizado para el programa de cálculo de pérdidas y reconfiguración topológica desarrollado en MATLAB. 
Tabla 3. Comparación de resultados de pérdidas de energía.

\begin{tabular}{cccc}
\hline Pérdidas de energía semanal (kWh) \\
Circuito & $\begin{array}{c}\text { Calculadas } \\
\text { cada 15 } \\
\text { minutos }\end{array}$ & $\begin{array}{c}\text { Calculadas } \\
\text { ecuaciones 1,2 } \\
\mathbf{y} 3\end{array}$ & $\begin{array}{c}\text { Porcentaje } \\
\text { de error }\end{array}$ \\
\hline EL & 153,9 & 149,4 & 2,94 \\
FA & 749,2 & 763,5 & 1,91 \\
SJ & 9268,2 & 9470,4 & 2,18 \\
IA & 5100,7 & 4912,2 & 3,69 \\
IN & 3341,6 & 3365,9 & 0,73 \\
AS & 4549,3 & 4553,3 & 0,09 \\
PA & 4886,2 & 4965,2 & 1,62 \\
MU & 1439,7 & 1410,1 & 2,06 \\
SN & 7307,0 & 7259,4 & 0,65 \\
TR & 1304,3 & 1261,1 & 3,31 \\
BA & 6115,4 & 6251,3 & 2,22 \\
GU & 3917,7 & 3886,9 & 0,79 \\
RA & 4361,8 & 4450,7 & 2,04 \\
PU & 3695,6 & 3653,4 & 1,14 \\
LL & 8319,8 & 8221,9 & 1,18 \\
SL & 10085,1 & 10631,9 & 5,42 \\
\hline
\end{tabular}

\subsection{Pérdidas en los circuitos de la CNFL}

Para lograr una adecuada caracterización de los circuitos de media tensión, se analizaron detalladamente las curvas de demanda semanal de 16 circuitos seleccionados mediante muestreo aleatorio. Utilizando el software de simulación de sistemas de potencia CYMDIST, se determinaron las pérdidas de cada uno de los periodos (de 15 minutos). Los resultados obtenidos se muestran en la Tabla 1.

\subsection{Constante del factor de pérdidas}

A partir de los resultados obtenidos en la sección anterior se puede obtener el factor de pérdidas (FP, Ecuación 2) y el factor de carga (FC) de cada circuito y con éstos, el factor A como sigue.

$A=\left(F P-F C^{2}\right) /\left(F P-F C^{2}\right)$

En la Tabla 2 se muestran los resultados para los circuitos analizados. 
De acuerdo a los resultados mostrados, el valor de la constante A para los alimentadores de la CNFL es 0,319. Para esta constante, la varianza obtenida es de 0,0028 lo que implica que para un nivel de confianza de $95 \%$ el error de esta constante es menor a $2,3 \%$.

Se puede afirmar entonces que para el cálculo de pérdidas en los alimentadores de la CNFL es más conveniente adoptar 0,319 para la constante $\mathrm{A}$ antes que cualquier otro valor recomendado por otros autores.

\subsection{Verificación del uso de la constante determinada}

En la Tabla 3 se muestran los resultados de obtención de las pérdidas mediante el cálculo de periodos cada 15 minutos y las determinadas mediante las Ecuaciones 1, 2 y 3, utilizando la constante A determinada.

Con estos resultados se valida la utilización de las ecuaciones para calcular las pérdidas en los alimentadores y para encontrar una topología de media tensión que reduzca las mismas.

\section{RECONFIGURACIÓN TOPOLÓGICA DE SISTEMAS DE MEDIA TENSIÓN}

La reconfiguración topológica de la red de distribución consiste en encontrar la estructura de operación radial que minimice las pérdidas de energía activa. Para esta reducción no se consideran las pérdidas en los transformadores de distribución ni en los circuitos secundarios, ya que las mismas no dependen de la configuración de la red de media tensión y se mantienen sin importar desde donde sea alimentado el transformador.

Existen distintos métodos que han sido utilizados para reconfiguración topológica de sistemas de distribución. Estos se dividen en los que utilizan métodos basados en conocimiento (heurísticos, programación lineal, lógica difusa), los que son basados en técnicas evolutivas (algoritmos genéticos, redes neuronales) y los métodos mixtos, siendo los métodos heurísticos los más utilizados por su simplicidad, esto según Caicedo et all
(2002); González, Hifikepunje y Lisan (2011); Reddy y Sydulu (2007); Savier \& Das (2007); Veera, Perumal \& Rajasekharareddy (2004); Zhu1, Xiong, Hwang, \& Sadjadpourl (2007).

El problema de la reconfiguración topológica, de acuerdo a González et all (2011), consiste en proporcionar un sistema de distribución conformado por alimentadores radiales interconectados entre sí en uno o más puntos y determinar el estado de todos los interruptores (abierto o cerrado) que minimicen las pérdidas de energía, contemplando los límites de tensión y capacidad de trasiego de potencia de cada tramo. Debido a la naturaleza combinatoria del problema, se requiere revisar una gran cantidad de posibilidades en casos reales (González et all, 2011). Existe además de los límites mencionados, la condición operativa de la red de distribución, que requiere maximizar la cantidad de enlaces y posibles respaldos de una red (o al menos mantener los que se tengan antes de hacer el análisis), esto normalmente basado en las experiencias de la operación del sistema de Zhu1, Xiong, Hwang, \& Sadjadpourl (2007).

Basado en estos argumentos, se plantea la elaboración de un programa que utilice el método heurístico y que tome en cuenta estos requerimientos operativos para determinar la configuración que minimice las pérdidas de energía activa.

\section{PROGRAMA PARA LA RECONFIGURACIÓN TOPOLÓGICA}

El programa desarrollado en MATLAB, parte de la lectura de tablas que contienen la información de un sistema de distribución de media tensión: cantidad de tramos y nodos, resistencia de cada tramo, características de las cargas conectadas, posición de los nodos, interruptores y acciones posibles. La tabla de acciones posibles se refiere a cada pareja de interruptores que pueden ser accionados (abrir uno y cerrar el otro) sin reducir las posibilidades de enlaces entre circuitos existentes actualmente.

A partir de los datos leídos de las tablas, el programa determina la cantidad de topologías 


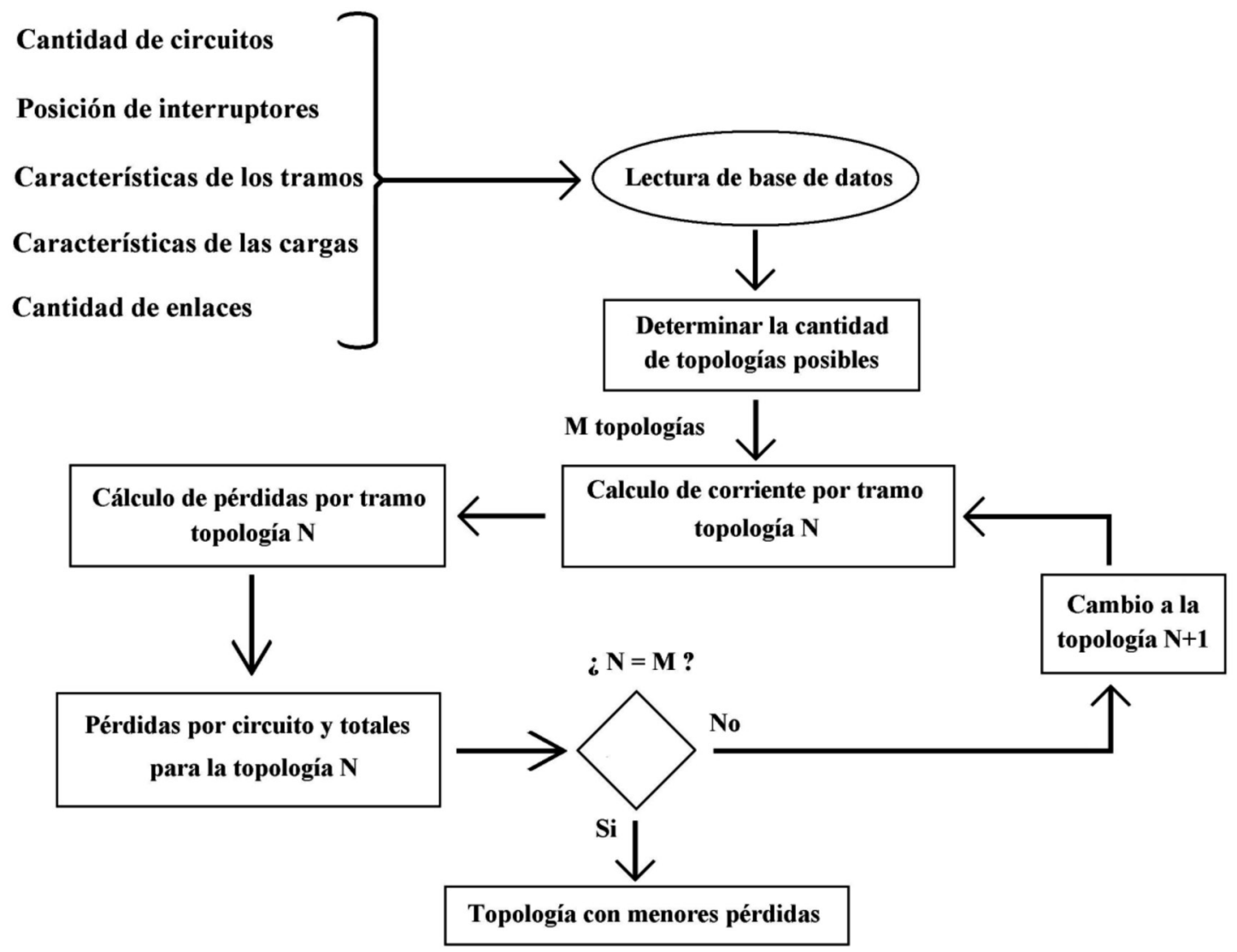

Figura 1. Diagrama de bloques del programa desarrollado.

posibles y para cada una calcula la corriente en cada tramo y con eso las pérdidas de energía por tramo, por circuito y el total de la zona en estudio. Para cada topología analizada, guarda en un vector los resultados de pérdidas de energía por circuito y pérdidas totales de esa configuración. Al finalizar la simulación, el programa indica cual es la topología con las menores pérdidas de energía. Este proceso se muestra en el diagrama de bloques de la Figura 1.

Los supuestos realizados para la elaboración del programa son los siguientes:

- Todas las cargas (transformadores de distribución) de un circuito tienen el mismo perfil de demanda que el circuito al que se encuentran conectadas. Para poder eliminar este supuesto, se requeriría tener medición en cada uno de los transformadores de distribución, condición que no se tiene actualmente.

- El nivel de tensión es 1 pu a lo largo de todo el circuito, esto es, la corriente en cada una de las cargas es determinada a partir de la demanda (valor conocido) dividiéndola por la tensión nominal del sistema. Este supuesto es totalmente válido en sistemas con circuitos de corta longitud.

- El programa determina las pérdidas para la máxima demanda y obtiene las pérdidas de energía por año aplicando las Ecuaciones 1 y 2.

- Para determinar la cantidad posible de topologías de un sistema, se requiere como parte de las entradas, una tabla que indique las 


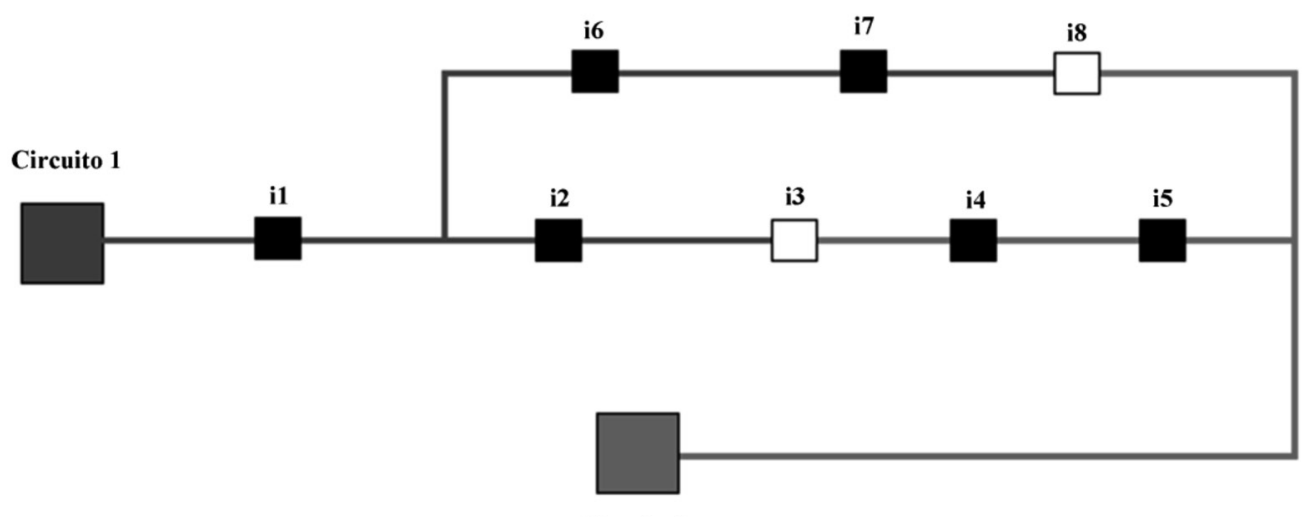

Circuito 2

Figura 2. Cantidad posible de topologías.

Tabla 4. Posibles cambios topológicos.

\begin{tabular}{ccc}
\hline \multirow{2}{*}{ Posibilidad } & \multicolumn{2}{c}{ Número de cuchilla o interruptor } \\
\cline { 2 - 3 } & Cerrar & Abrir \\
\hline 1 & i8 & i7 \\
2 & i8 & i6 \\
3 & i3 & i2 \\
4 & i3 & i4 \\
5 & i3 & i5 \\
\hline
\end{tabular}

posibles combinaciones de cada enlace, por ejemplo, para un sistema conformado por dos circuitos como el de la Figura 2, el programa requiere la información de cada enlace, básicamente como se muestra en la Tabla 4.

Nótese que la operación de interruptor i1 reduce la cantidad de enlaces entre el circuito 1 y el circuito 2 por lo que no es tomado en cuenta como opción.

A pesar de tener 5 posibles operaciones, las posibilidades 1 y 2 no son compatibles entre sí, al igual que las posibilidades 3,4 y 5 por lo que para este sistema se tendrían en total 6 posibles topologías para analizar.
La CNFL cuenta con un Sistema de Información Geográfica (SIGEL) el cual contiene la información de todos los elementos de la red de distribución en forma tabulada. Estas tablas son las utilizadas por el programa desarrollado en MATLAB como base de datos para las simulaciones. La información contenida en las tablas es: identificador de sección, circuito, nodo de salida, nodo de llegada, número y tipo de dispositivo, identificador de línea, identificador de cable, longitud del tramo, carga en la fase A, carga en la fase B y carga en la fase C.

El programa calcula la corriente en cada una de las cargas y con eso la corriente en cada uno 
Tabla 5. Comparación de resultados de pérdidas en demanda máxima.

\begin{tabular}{cccc}
\hline Circuito & MATLAB & $\begin{array}{c}\text { Pérdidas }(W) \\
\text { CYMDIST }\end{array}$ & \% error \\
\hline CC & 13533 & 13770 & 1,72 \\
RA & 56394 & 56350 & 0,08 \\
SS & 5790 & 5840 & 0,86 \\
E1 & 8946 & 8900 & 0,52 \\
CR & 40856 & 41320 & 1,12 \\
FA & 9150 & 9120 & 0,33 \\
\hline
\end{tabular}

de los tramos de cada circuito. Posteriormente, determina las pérdidas de energía en cada tramo y realiza la suma para determinar las pérdidas de energía totales en el circuito, todo por fase.

Las pérdidas determinadas por el programa fueron verificadas con las obtenidas con el Software CYMDIST. La comparación de resultados para algunos circuitos se muestra en la Tabla 5 .

Como se puede apreciar, los resultados obtenidos con el programa elaborado en MATLAB son prácticamente los mismos que con el software de simulación de sistemas de distribución CYMDIST lo que valida los supuestos que utiliza el programa desarrollado.

\subsection{Sistema de media tensión analizado}

La comprobación del funcionamiento de los cambios topológicos del programa desarrollado se hizo en la zona oeste de la red de media tensión del la CNFL, $(34,5 \mathrm{kV})$ que está compuesta por 5 subestaciones y un total de 35 circuitos. Estas subestaciones están enlazadas entre sí en más de un punto, lo que permite una considerable cantidad de posibles topologías que mantienen la cantidad de enlaces y posibilidades operativas actuales.

Para el análisis se tuvieron algunas consideraciones propias de los circuitos analizados y la zona que atienden: los circuitos de generación o mixtos no se toman en cuenta (3 circuitos), los circuitos sin enlaces que cumplan con lo establecido en los criterios no se consideran (5 circuitos), los enlaces con otras subestaciones se consideran como colas de circuito.

De acuerdo a las consideraciones tomadas en cuenta, es posible dividir el área de implementación del programa en tres zonas independientes entre si (sin enlaces) por lo que en adelante se tratarán por separado. En este artículo se detallan los resultados obtenidos para la Zona 1, los de la Zona 2 y 3 solo se mencionan. En total, el sistema que se analiza está compuesto por 20 circuitos divididos como sigue:

- Zona 1: 5 circuitos

- Zona 2: 6 circuitos

- Zona 3: 9 circuitos

En la Tabla 6 se muestra la demanda máxima, factor de carga y de pérdidas para los circuitos de la zona 1 y el promedio de todos los analizados.

\subsection{Aplicación del programa}

Como se mencionó, de acuerdo a las consideraciones realizadas, se dividió el área de implementación del programa en tres. A continuación se detallan las características de cada área y los principales resultados obtenidos en cada una. 
Tabla 6. Demanda máxima de los circuitos incluidos en el análisis.

\begin{tabular}{|c|c|c|c|c|}
\hline Subestación & Circuito & $\begin{array}{c}\text { Demanda } \\
\text { máxima (MW) }\end{array}$ & Factor de carga & $\begin{array}{c}\text { Factor de } \\
\text { pérdica }\end{array}$ \\
\hline \multirow{2}{*}{ Sub1 } & SN & 10,11 & 0,694 & 0,5494 \\
\hline & SS & 3,37 & 0,667 & 0,5158 \\
\hline Sub2 & RA & 12,96 & 0,657 & 0,5035 \\
\hline \multirow{2}{*}{ Sub3 } & FO & 1,36 & 0,672 & 0,5218 \\
\hline & $\mathrm{CC}$ & 4,60 & 0,687 & 0,5406 \\
\hline \multicolumn{2}{|c|}{ Promedio } & 7,93 & 0,702 & 0,5616 \\
\hline
\end{tabular}

\subsubsection{Zona 1}

La Figura 3 muestra el diagrama unifilar de los cinco circuitos que conforman la Zona 1.

Para esta zona, existen 8 posibilidades de cierre y apertura de cuchillas o interruptores bajo los criterios ya establecidos. En la Tabla 7 se muestran esas posibilidades.

Para esas 8 posibilidades de cierre y apertura, existen 108 combinaciones posibles (considerando la configuración actual que se toma como la número uno). Esta cantidad, fue determinada por el programa desarrollado.

Una vez que se ejecuta el programa, realiza los cálculos para las 108 topologías y para cada una entrega las salidas:

- Pérdidas en demanda máxima para cada circuito.

- Pérdidas de energía anual para cada circuito y el total para cada topología.

- Configuración de cada topología (figura con referencia geográfica).

- Topología con las menores pérdidas de energía en media tensión.

Adicionalmente, el programa podría entregar datos como la corriente o potencia por fase en la fuente de cada circuito, estado de cada interruptor para una topología determinada, entre otros.
En el caso de los cinco circuitos en cuestión, la primera topología calculada por el programa es la actual (ver Figura 3). Para este caso, la salida del programa es la que se aprecia en la Figura 4.

Adicionalmente el programa entrega el mapa de la configuración como se muestra en la Figura 5.

De la misma manera, se obtiene los resultados para las otras 107 topologías. A modo de ejemplo, en la Figura 6 se muestran los resultados para 4 topologías.

Al finalizar la simulación el programa indica cual es la topología con las menores pérdidas, en este caso, la topología 107, tal como se muestra en la Figura 7.

En la Zona 1, 49 de las 108 topologías analizadas presentan pérdidas inferiores a las actuales. El número de topología corresponde al número de fila de la tabla de posibles combinaciones que el mismo programa creo. En este caso, la fila 107 corresponde al cierre de los interruptores o cuchillas $8008,8006,8077,8067$ y 8041 y la apertura de los interruptores o cuchillas números 8053, 8010, 8082, 8043 y 8003 (ver figura 3). Esta topología se muestra gráficamente en la Figura 8.

Para esta topología, las pérdidas de energía en la red de media tensión se reducen en aproximadamente 144,26 MWh por año con respecto a la topología actual. La demanda 


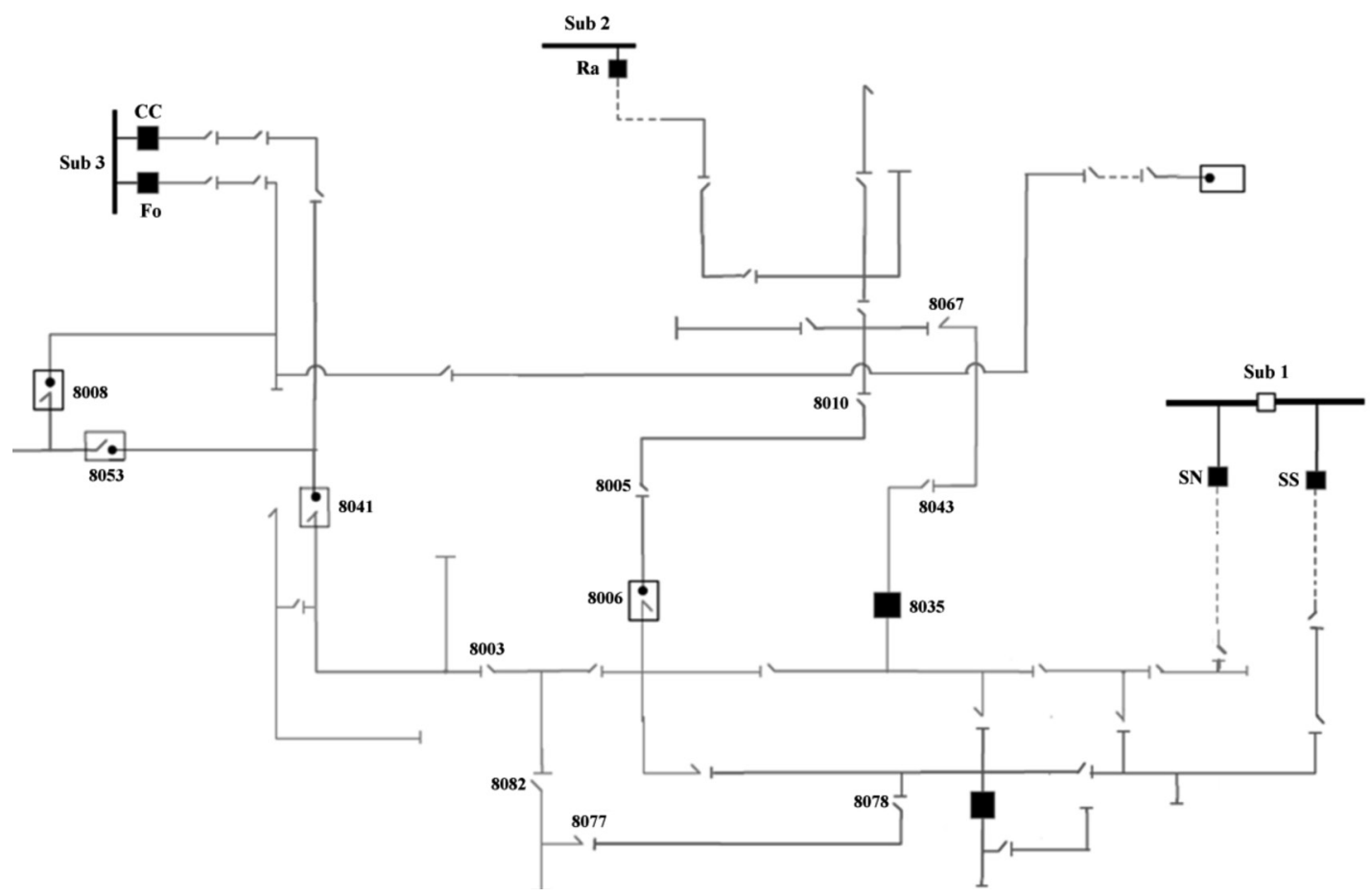

Figura 3. Diagrama Unifilar de la Zona 1.

Tabla 7. Posibles cambios topológicos - Zona 1.

\begin{tabular}{ccc}
\hline \multirow{2}{*}{ Posibilidad } & \multicolumn{2}{c}{ Número de cuchilla o interruptor } \\
& Cerrar & Abrir \\
\cline { 2 - 3 } 1 & 8008 & 8053 \\
2 & 8006 & 8005 \\
3 & 8006 & 8010 \\
4 & 8077 & 8078 \\
5 & 8077 & 8082 \\
6 & 8067 & 8043 \\
7 & 8067 & 8035 \\
8 & 8041 & 8003 \\
\hline
\end{tabular}




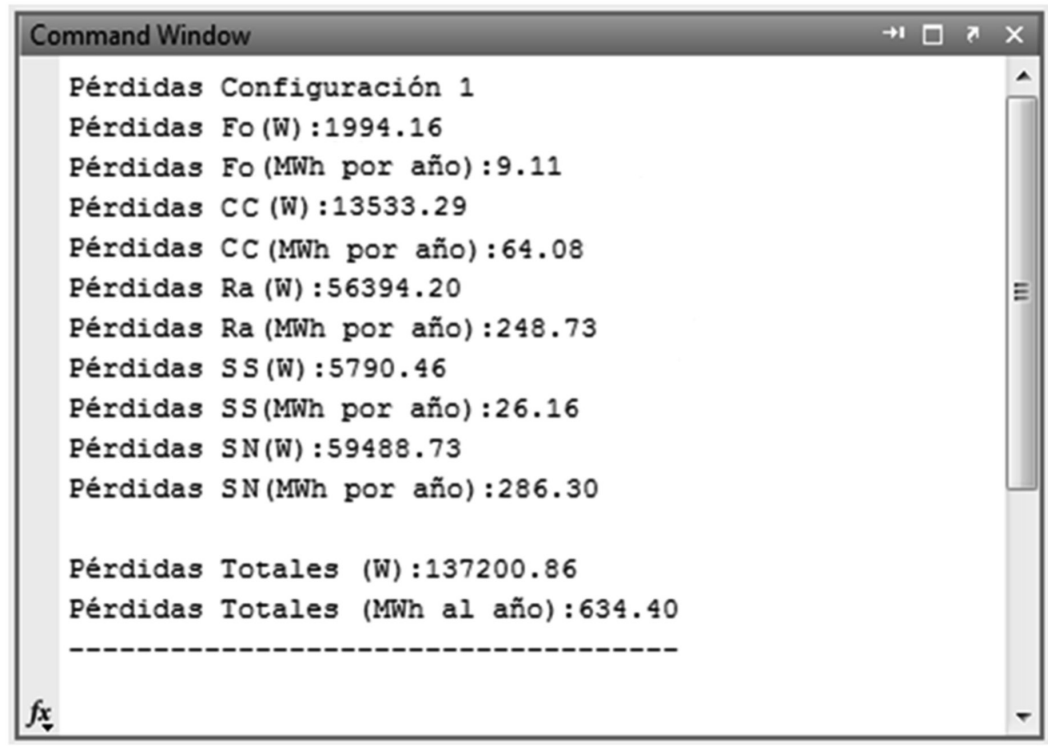

Figura 4. Resultados para la Configuración 1.

máxima y pérdidas por circuito de la nueva topología y de la actual se muestran en la Tabla 8.

\subsubsection{Zona 2}

Esta zona está compuesta por 6 circuitos y cuenta con 540 topologías, de las cuales 23 presentan pérdidas inferiores a las de la actual. Para la mejor topología, las pérdidas se reducen en 4,85 MWh por año con respecto a la topología actual.

\subsubsection{Zona 3}

Esta zona está compuesta por 9 circuitos de distribución y un total de 2220 topologías posibles bajo los criterios expuestos. En esta zona se obtuvieron 108 topologías con pérdidas menores a las actuales, representando la menor de ellas una reducción de 135,2 MWh por año.

\subsubsection{Reducción de pérdidas de energía}

En total, se evaluaron 129470400 topologías para la zona de estudio. En la Tabla 9 se muestran las pérdidas para la topología actual y para la de menores pérdidas de energía.

Se tiene entonces, una reducción en las pérdidas de energía de 284,3 MWh por año con la topología propuesta. Convertir esta cifra a valor económico no es una labor sencilla, debido al sistema tarifario donde a distintas horas del día se tiene un costo distinto la energía y adicionalmente existe un costo por la demanda máxima que por supuesto se vería reducida en algún porcentaje (muy bajo claro está). Un cálculo conservador sobre el valor económico de la reducción de pérdidas de energía puede realizarse utilizando la tarifa para el periodo nocturno (43,7 colones por kWh más 9,74 colones por kWh por el cargo de transmisión, tarifas publicadas el 2 de julio de 2013), con la cual se obtiene un ahorro cercano a los 15,2 millones de colones al año. Por otra parte, el cálculo de la reducción económica que se tendría por la reducción en la demanda máxima, no se puede hacer con las herramientas e información disponible debido a la diversidad en la hora de la demanda máxima de la CNFL y la demanda máxima de cada uno de los circuitos incluidos en el análisis.

Para poder implementar los resultados obtenidos se deben realizar aperturas y cierres 


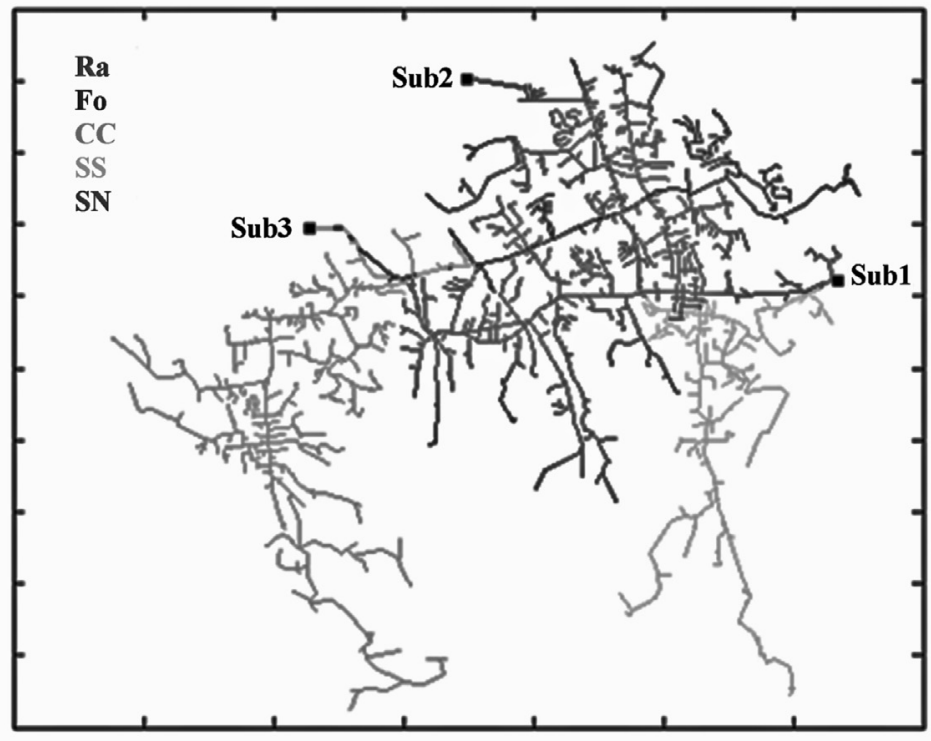

Figura 5. Diagrama de la topología actual - Zona 1.

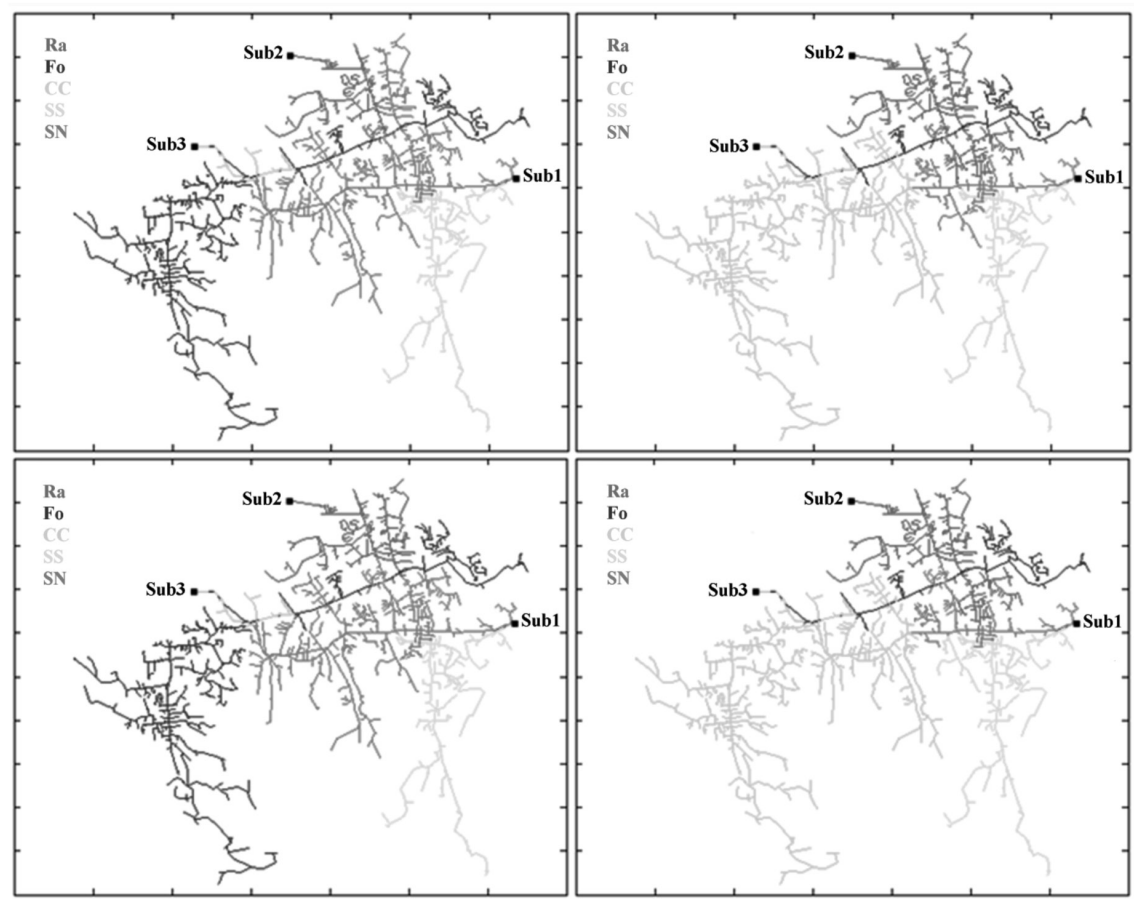

Figura 6. Ejemplo de topologías evaluadas Zona 1. 


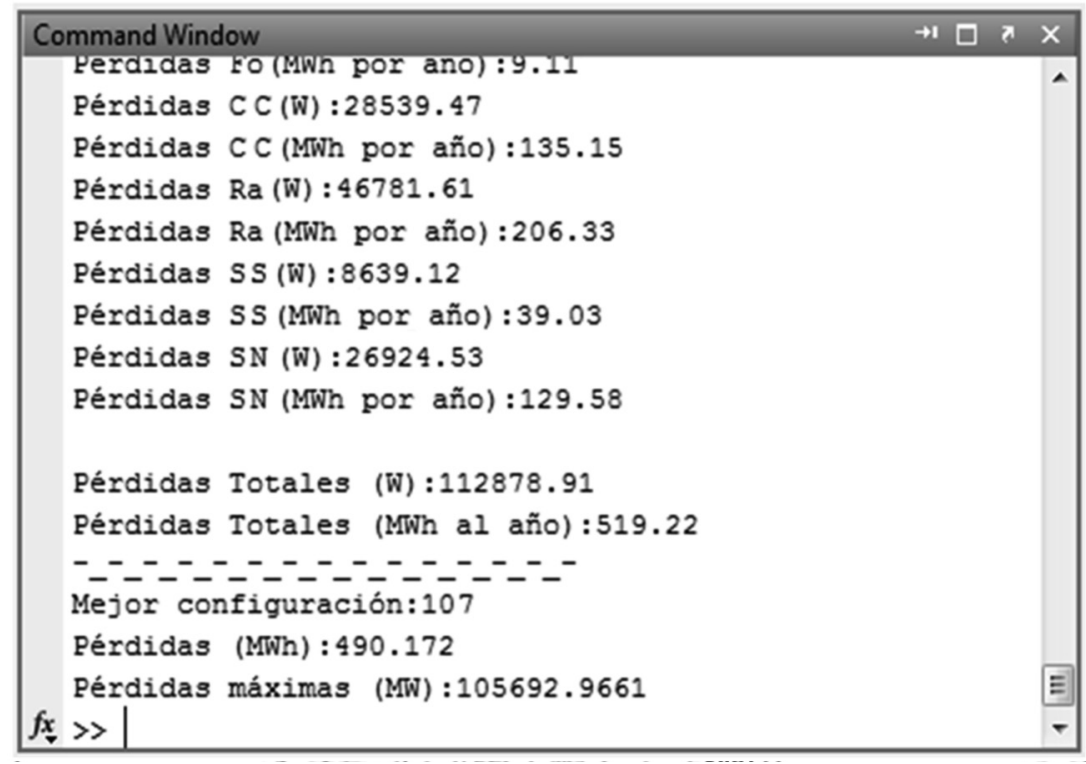

Figura 7. Resultado de las 108 topologías analizadas - Zona 1.

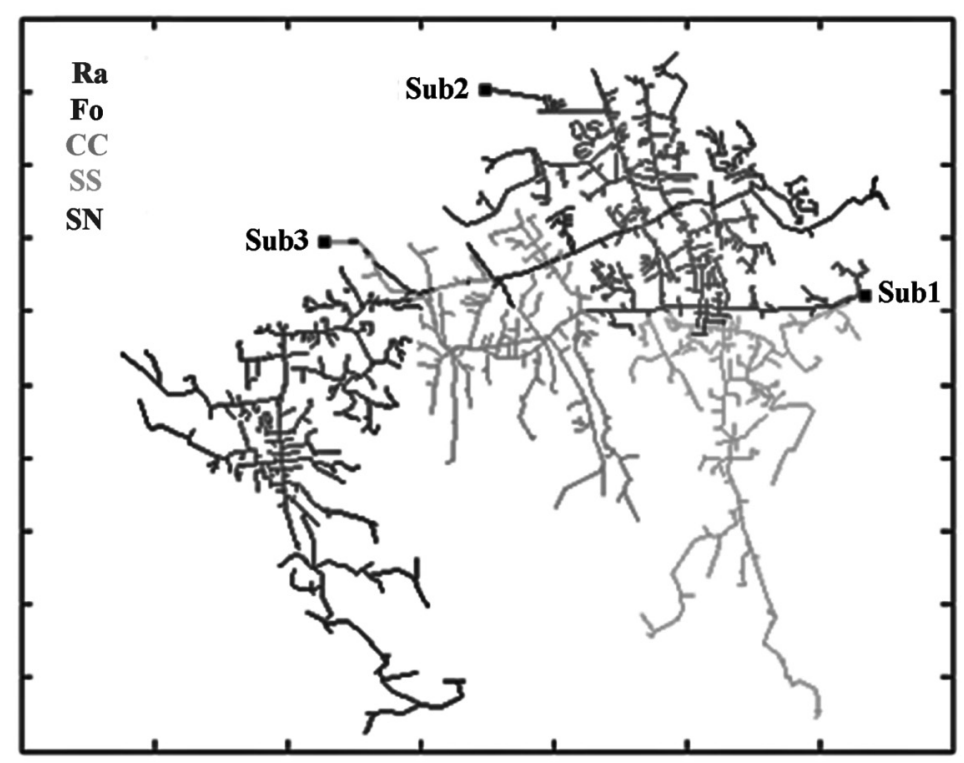

Figura 8. Topología con menores pérdidas de energía - Zona 1. 
Tabla 8. Demanda máxima y pérdidas por circuito.

\begin{tabular}{ccccc}
\hline \multirow{2}{*}{ Circuito } & \multicolumn{2}{c}{ Demanda máxima(MW) } & \multicolumn{2}{c}{ Pérdidas (MWh al año) } \\
& Actual & Propuesta & Actual & Propuesta \\
\hline FO & 1,36 & 5,12 & 9,12 & 70,62 \\
CC & 4,60 & 4,84 & 64,09 & 45,68 \\
RA & 12,96 & 9,23 & 248,73 & 125,91 \\
SS & 3,37 & 3,92 & 26,16 & 39,03 \\
SN & 10,11 & 9,33 & 286,30 & 208,90 \\
& & Total & 634,41 & 490,17 \\
\hline
\end{tabular}

Tabla 9. Reducción de pérdidas de energía.

\begin{tabular}{ccc}
\hline Zona & \multicolumn{2}{c}{ Pérdidas de energía anual(MWh) } \\
Actual & Topologías propuestas \\
\hline 1 & 634,41 & 490,17 \\
2 & 204,72 & 199,87 \\
3 & 2866,05 & 2730,80 \\
Total & 3705,18 & 3420,84 \\
\hline
\end{tabular}

de interruptores, seccionadores o cuchillas. En caso de que sean cuchillas, es una labor que requiere de una inversión baja en comparación con las ganancias o reducción de pérdidas. En el caso de que sean interruptores de enlace, se debe hacer un análisis económico para determinar la conveniencia de hacer los cambios, ya que los mismos deben ser intercambiados por cuchillas y ser instalados en finales de circuito para que puedan seguir funcionando de la manera que lo hacen actualmente. Para la mejor topología determinada, se encontró que 5 de los 24 puntos que deben cambiar su estado normal de operación (abierto o cerrado) son interruptores, pero dos de ellos cambian de estado entre sí por lo que solo se requeriría del intercambio de 3 equipos. Según datos brindados por la Sección de Diseño de Redes Eléctricas de la CNFL, el costo del cambio de un dispositivo por otro es cercano a los 300000 colones más transporte, lo que indica que es un cambio totalmente factible económicamente.

\subsection{Comprobación de resultados}

Para comprobar los resultados obtenidos con el programa desarrollado se realizaron las operaciones necesarias para obtener en CYMDIST la topología mostrada en la Figura 6, correspondiente a la de menores pérdidas de la Zona 1. La comparación de los resultados se muestra en la Tabla 10.

Como se aprecia, los resultados son prácticamente los mismos, las diferencias se deben a los supuestos realizados por el programa 
Tabla 10. Comprobación de la topología con menores pérdidas - Zona 1.

\begin{tabular}{cccc}
\hline \multirow{2}{*}{ Circuito } & \multicolumn{2}{c}{ Perdidas $(\mathbf{k W})$} & $\begin{array}{c}\text { Porcentaje de } \\
\text { error }\end{array}$ \\
\hline CYMDIST & MATLAB & $0,39 \%$ \\
CC & 15,51 & 15,45 & $1,03 \%$ \\
RA & 9,75 & 9,65 & $0,24 \%$ \\
SS & 28,62 & 28,55 & $0,69 \%$ \\
SN & 8,70 & 8,64 & $0,85 \%$ \\
Total & 43,77 & 43,40 & $0,62 \%$ \\
\hline
\end{tabular}

elaborado en MATLAB. De esta manera, quedan validados los resultados que se obtienen con el programa desarrollado.

\section{CONCLUSIONES Y RECOMENDACIONES}

\subsection{Conclusiones}

- El factor de carga de los circuitos de la zona oeste de CNFL es en promedio 0,70 , mientras que el factor de pérdidas es aproximadamente 0,56 . El muestreo realizado para estos valores permitió obtener con suficiente confianza, el valor de la constante que los relaciona es A $=0,319$. Con esto se deja de utilizar el valor recomendado por autores de otros países y se implementa el valor que más se ajusta a las condiciones de carga de la CNFL.

- Se demostró que el error en el que se incurre al calcular las pérdidas de energía utilizando las pérdidas en demanda máxima y el Factor de Pérdidas es en promedio 0,03\%, justificando con esto su utilización en el programa desarrollado y en futuros estudios técnicos y económicos para justificación de obras por ejemplo.
- Las pérdidas en la red de distribución de media tensión representan en promedio $0,45 \%$ de la demanda máxima de cada circuito y las de energía son aproximadamente $0,35 \%$ de la total trasegada por el circuito en un periodo de tiempo dado.

- El factor de uso de los circuitos de media tensión analizados, que se define como la demanda máxima entre la capacidad instalada del alimentador es en promedio 0,32, lo que sugiere una gran capacidad instalada que no es utilizada (transformadores de distribución subutilizados). Este hecho es conocido por las empresas distribuidoras, sin embargo, pocas veces se cuantifica a partir de una muestra como la analizada en este proyecto (26 circuitos de media tensión).

- Se desarrolló un programa en MATLAB que determina las pérdidas en los circuitos de media tensión a partir de bases de datos ya existentes y se comprobaron los resultados, obteniendo errores inferiores a $2 \%$ lo que se considera aceptable para los fines del mismo.

- El programa desarrollado determina a partir de datos de interruptores, seccionadores o cuchillas que pueden cambiar su estado la cantidad de combinaciones posibles. En el caso de la zona oeste compuesta por cinco subestaciones, se determinaron $129 \quad 470$ 
400 combinaciones posibles, todas estas manteniendo la cantidad de enlaces existentes.

- El programa desarrollado muestra de manera gráfica las topologías evaluadas, permitiendo con esto la fácil visualización de los resultados y una más rápida interpretación de los mismos.

- El programa realizó la evaluación de las 129470400 topologías (divididas en tres zonas) y determinó la combinación que tiene las menores pérdidas en media tensión, concluyendo en una reducción cercana a 285 MWh por año mediante el cambio de estado de 5 interruptores y 19 cuchillas.

- La implementación de los resultados obtenidos es económicamente viable pues la inversión para realizar la mayoría de ellos (excepto los que requieren el cambio de interruptor por cuchillas y viceversa) es despreciable en comparación con la disminución de las pérdidas de energía que se obtendrá.

- Las pérdidas obtenidas para la topología con las menores pérdidas en media tensión, fueron comparadas con las obtenidas con CYMDIS, obteniendo porcentajes de error despreciables, en promedio $0,62 \%$, validando con esto los resultados obtenidos por el programa desarrollado.

\subsection{Recomendaciones}

- Extender el análisis realizado a toda el área de distribución de la CNFL, incluyendo la red aérea de $13,8 \mathrm{kV}$ de tensión nominal y el sistema de distribución subterránea de San José.

- Realizar los análisis operativos necesarios, in cluyendo el de balance entre fases, para confirmar la viabilidad de la implementación de la topología propuesta con el fin de reducir las pérdidas de energía en media tensión.

- Realizar las investigaciones necesarias para determinar la viabilidad de realizar un programa similar en la plataforma Visual. net con el fin de que pueda ser implementado directamente con SIGEL y con esto reducir o incluso eliminar los tiempos de preparación de la base de datos.
- Agregar al programa restricciones que permitan descartar topologías antes de ser calculadas las pérdidas, por ejemplo, descartar los casos en los que hayan circuitos con demandas máximas superiores a algún nivel predeterminado. Esto permitiría reducir apreciablemente las posibilidades y con esto los tiempos de simulación y solución.

- Elaborar programas o módulos similares al desarrollado que determinen el perfil de tensión y evalúen la confiabilidad de cada topología con el fin de crear una serie de programas que conlleven a la determinación de la topología óptima de la red de distribución.

- Realizar las simulaciones en el programa desarrollado con demandas proyectadas a 5 y 10 años (o incluso intervalos menores si se considera necesario) y analizar los posibles cambios que se den en la topología con menores pérdidas y realizar el análisis económico que incluya la posible inversión que se requiera para los cambios topológicos (cambio de interruptores y cuchillas por ejemplo).

\section{NOTAS}

1. Tomando como valor real el obtenido con CYMDIST.

\section{BIBLIOGRAFÍA}

Caicedo, N.G., Lozano, C.A., Díaz, J.F., Rueda, C., Gutiérrez, G. \& Olarte, C. (2002). Loss Reduction in Distribution Networks using Concurrent Constraint Programming, Colombian National Research Support Institute and EPSA.

Fernández Vásquez, R. (2007). Determinación y proyección de los niveles de cortocircuito en el sistema de distribución de la CNFL hasta el 2015, Proyecto de Licenciatura para la obtención de título de Licenciado en Ingeniería Eléctrica, Facultad de Ingeniería, Universidad de Costa Rica, Costa Rica. 
González, J.A., Hifikepunje, J. \& Lisan, I. (2011). Reconfiguración de Sistemas de Distribución Mediante Intercambio de Ramas, CIE Cuba.

Kersting, W.H. (2000). Distribution System Modeling and Analysis. Electric Power Engineering Series, Estados Unidos.

Reddy, V.V.K., Sydulu, M. (2007). A HeuristicExpert based Approach for Reconfiguration of Distribution Systems, Ponencia presentada en IEEE PES General Meeting, Tampa.

Savier, J. S. \& Das, D. (2007). Impact of Network Reconfiguration on Loss Allocation of Radial Distribution Systems. IEEE Transactions on Power Delivery, vol. 22, no. 4, 2473-2480.

Short, T.A. (2004). Electric Power Distribution Handbook. Florida, USA.

Veera, V.C., Perumal, N. \& Rajasekharareddy, Y. (2004). Loss Reduction in Distribution Networks by Network Reconfiguration: A two Stage Solution Approach. National
Power \& Energy Conference. Proceedings, Kuala Lumpur, Malaysia, pp.241-246.

Zhu1, J., Xiong, X., Hwang, D. \& Sadjadpourl, A. (2007). A Comprehensive Method for Reconfiguration of Electrical Distribution network, IEEE PES General Meeting, Tampa.

\section{SOBRE EL AUTOR}

\section{Raúl Fernández Vásquez}

Máster en Ingeniería Eléctrica con énfasis en Sistemas de Mediana y Baja Tensión en la Universidad de Costa Rica. Departamento de Planificación y Diseño de la Compañía Nacional de Fuerza y Luz, S.A., Costa Rica.

Correo electrónico: rfernandez@cnfl.com. Teléfonos (506) 2295-5683, Fax (506) 2295-5684. Apartado Postal 10026-1000. 
